\title{
UNIVERSIDADE EMPREENDEDORA E TRANSFERÊNCIA DE CONHECIMENTO E TECNOLOGIA
}

\author{
Barbara Kobuszewski Volles* \\ barbarakvolles@gmail.com \\ Giancarlo Gomes* \\ giancarlog@furb.br
}

Iara Regina dos Santos Parisotto*

iaraparisotto@furb.br

*Universidade Regional de Blumenau - SC / Brasil

http://dx.doi.org/10.1590/1413-2311.03716.61355

Recebido em 07/01/2016

Aprovado em 02/05/2017

Disponibilizado em 31/05/2017

Avaliado pelo sistema "double blind review"

Revista Eletrônica de Administração

Editora-chefe: Andrea Oltramari

ISSN 1413-2311 (versão "on line")

Editada pela Escola de Administração da Universidade do Rio Grande do Sul

Periodicidade: Quadrimestral

Sistema requerido: Adobe Acrobat Reader

\section{RESUMO}

Este estudo tem como objetivo analisar a influência da universidade empreendedora formada por diferentes dimensões no contexto de uma universidade. O objeto de estudo selecionado foram estudantes de graduação uma instituição de ensino superior de Santa Catarina. Os resultados encontrados comprovam que as dimensões da universidade empreendedora: mobilização de pesquisa, colaboração da indústria, informalidades e interação das indústrias formam a universidade empreendedora, bem como influenciam nas atividades empreendedoras da mesma. A universidade estudada apresenta baixo nível de atividade empreendedora, comprovando a partir do modelo a necessidade de diferentes indicadores para que uma universidade seja empreendedora. Sugere-se então a reaplicação do modelo em outras universidades para que haja uma verificação da intensidade das atividades empreendedoras em outras instituições de ensino superior. 
Palavra-chave: Universidade Empreendedora. Orientação Empreendedora. Conhecimento, Tecnologia.

\title{
ENTREPRENEURIAL UNIVERSITY AND TRANSFER OF KNOWLEDGE AND TECHNOLOGY
}

\begin{abstract}
This study aims to analyze the influence of entrepreneurial University formed by different dimensions in the context of a University. The study object was graduate students of a University in Santa Catarina. The results show that the dimensions of the entrepreneurial university: mobilizing research, industry collaboration, informalities and industries interaction, form the entrepreneurial University, as well as influence the entrepreneurial activities of high education institutions. The studied university has a low level of entrepreneurial activity, demonstrating from the explored model the need for different indicators for a university to be entrepreneurial. It is suggested the model reapplication in different universities, in order to check entrepreneurial activities in other higher education institutions.
\end{abstract}

Keywords: Entrepreneurial University. Entrepreneurial Orientation. Knowledge; Tecnology.

\section{INTRODUÇÃO}

A universidade empreendedora é aquela que apresenta a habilidade de inovar e criar oportunidades; trabalhar em equipes, assumir riscos e responder a desafios (GUERRERO e URBANO, 2012). Audretsch (2014) pontua que a universidade empreendedora tem a capacidade de disseminar a transferência de tecnologia baseada no conhecimento auxiliando nos esforços empresariais e influenciando uma sociedade a ser mais empreendedora. Logo, esta transferência de conhecimento e tecnologia ocorre pela implementação de diferentes estratégias incorporadas por diferentes instituições como empresas e governos (LEYDESDORFF e MEYER, 2014).

Sbragia (2006) explica que a necessidade da inclusão da inovação nos arranjos institucionais levou a fomentação de programas de incentivos às parcerias, fazendo com que houvesse uma maior interação entre Universidade e empresa. Porém, ainda existem barreiras 
que dificultam esta relação e fazem com que haja um lento desenvolvimento para que estas universidades se tornem empreendedoras (MANCINI e LORENZO, 2006; SBRAGIA, 2006; IPIRANGA et al. 2010). Apesar disso, os incentivos às parcerias Universidade-Empresa geram positivos impactos como o desenvolvimento de recursos humanos, criação de novos conhecimentos assim como sua comercialização por meio de divulgação de novas invenções, patentes, licenças, spin offs (ETZKOWITZ et al. 2000).

A orientação empreendedora nas Universidades vem sendo estudada por diferentes autores, tendo como principal objetivo o entendimento da transferência de tecnologia e conhecimento entre universidade-empresa (ETZKOWITZ, 1998, SHANE, 2002; WRIGHT et al., 2006; GUERRERO e URBANO, 2012; GUERRERO et al. 2014). Partes dos estudos referentes às atividades empreendedoras nas universidades vêm sendo praticados na Europa e Estados Unidos (MARKMANN et. al., 2005, ROTHERMEL et. al. 2007, PHILPOTT et al., 2011, GUERRERO et al. 2015).

Embora estas relações têm sido frequentemente estudadas há pouca evidência empírica no contexto nacional. Assim, este estudo busca preencher esta lacuna analisando a influência da Universidade Empreendedora formada pelas dimensões de Mobilização de Pesquisa, Colaboração da Indústria, Informalidades e Interação das Indústrias nas Atividades Empreendedoras no contexto de uma Universidade de Santa Catarina. Esta pesquisa justificase uma vez que tem potencial de apoiar os esforços da administração da universidade para avaliar a cultura de departamentos universitários e desenvolver condições mais favoráveis para os resultados de comercialização, tais como spin outs, patenteamento e licenciamento de inovações (TORODOVIC; MCNAUGHTON e GUILD, 2011). A pesquisa proporciona uma visão global do envolvimento dos acadêmicos em diferentes atividades de seus centros universitários, e como este percebe a universidade empreendedora.

$\mathrm{O}$ estudo encontra-se estruturado em cinco seções. A primeira seção corresponde a introdução do artigo, sendo compreendida pela apresentação do tema de pesquisa e objetivo a ser trabalhado. A segunda seção trata do modelo teórico e hipóteses. Na terceira seção é estruturada o método da pesquisa. Posteriormente, na quarta seção têm-se a análise dos dados. $\mathrm{Na}$ quinta seção as considerações finais a partir dos resultados demonstrados pela presente pesquisa e, por fim, as referências bibliográficas.

\section{MODELO TEÓRICO E HIPÓTESES}


Covin e Slevin (1989) pontuam que a Universidade Empreendedora está conectada a uma combinação de diferentes construtos formada pela inovação, pró-atividade e comportamento de riscos de uma organização. O constructo da Universidade Empreendedora se dá pela troca do ambiente acadêmico conservador, por um ambiente originador de conhecimento que integra crescimento econômico e desenvolvimento social às práticas de pesquisa e ensino (ETZKOWITZ e ZHOU, 2008; ETZKOWITZ, 2013).

Com base na revisão de literatura, são descritos na sequência as dimensões de primeira ordem - Mobilização de Pesquisa, a Colaboração da Indústria, as Informalidades e Interação das Indústrias, que formam o constructo de segunda ordem da Universidade Empreendedora, o qual influencia as Atividades Empreendedoras da universidade (KALAR e ANTONCIC, 2015; TORODOVIC; MCNAUGHTON e GUILD, 2011).

A Mobilização de Pesquisa segundo Kalar e Antoncic (2015) e Torodovic, Mcnaughton e Guild (2011) se refere ao envolvimento da universidade e o centro acadêmico no encorajamento dos seus alunos à pesquisa e à interação com as empresas e a sociedade. Com o objetivo de influenciar a cultura empreendedora a maioria das universidades adotam novas estruturas organizacionais e políticas de incentivo, para sensibilizar os alunos e funcionários, como professores de empreendedorismo especializados na área e cursos de empreendedorismo, incorporando às práticas empresariais (O’SHEA et. al., 2005; TIJSEN, 2006).

Portanto, a atividade de ensino, pesquisa e atividades empreendedoras, acabam influenciando o desenvolvimento econômico e social, contribuindo também para o surgimento de novos negócios (O’SHEA et al., 2005; TIJSEN 2006; GUERRERO et. al., 2015). Ripper Filho (1994) explica que estas vantagens devem estar ligadas aos objetivos básicos da Universidade e da empresa, ou seja, a universidade deve continuar contribuindo para a sua missão de formar recursos humanos e a empresa deve notar nela uma contribuição direta ou indireta para sua lucratividade. Nesse sentido levanta-se a seguinte hipótese de pesquisa: H1: A Mobilização de Pesquisa tem um efeito positivo na Universidade Empreendedora.

A Colaboração da Indústria é representada pelo reconhecimento das empresas referente ao envolvimento da Universidade em atividades de pesquisa com as empresas (KALAR e ANTONCIC, 2015; TORODOVIC; MCNAUGHTON e GUILD, 2011). No Brasil, a aproximação universidade-empresa limita-se ao acesso às competências que a empresa não possui e têm alto custo no mercado. Além disso, existem barreiras como a burocracia universitária, duração muito longa do projeto e diferenças de nível de 
conhecimento entre as pessoas da universidade e das empresas envolvidas na cooperação (COSTA E CUNHA, 2001; SEGATTO e SBRAGIA, 2002; BENEDETTI et al., 2011).

Tanto a cooperação entre universidade e empresa quanto à inovação tecnológica, ainda são práticas que precisam ser estimuladas e intensificadas, sendo esta uma característica comum em países em desenvolvimento (BENEDETTI et. al., 2011). Segatto e Sbragia (2002) apontam o fundo governamental de apoio à pesquisa um dos principais facilitadores da parceria, e uma vez iniciados a cooperação, as divergências entre as duas esferas são minimizadas pelo estreitamento do relacionamento, o que contribui para a velocidade da execução do projeto de desenvolvimento de uma nova tecnologia (BENEDETTI et al., 2011). Com base no exposto, tem-se a hipótese de pesquisa: H2: A Colaboração da Indústria tem um efeito positivo na Universidade Empreendedora.

As Informalidades estão conectadas a capacidade da universidade em buscar por oportunidades de atividades empreendedoras fora do âmbito acadêmico (KALAR e ANTONCIC, 2015; Torodovic, Mcnaughton e Guild; 2011) Uma Universidade que não demonstra estes interesses acabada desencorajando os estudantes a buscarem por novas oportunidades (RASMUSSEN, MOSEY e WRIGHT, 2014). O nível empreendedor do departamento é influenciado pelo acesso a parceiros comerciais e a legitimidade gerencial do departamento na criação de experiências comerciais e científicas (VAN LOOY et al., 2011; RASMUSSEN, MOSEY e WRIGHT, 2014). Também pode-se mencionar os incentivos governamentais por meio dos editais e programas como da Coordenação de Aperfeiçoamento de Pessoal de Nível Superior - CAPES e do Conselho Nacional de Desenvolvimento Científico e Tecnológico - CNPq, com o objetivo de incentivar as parcerias entre empresas e universidades (IPIRANGA, 2008; DA GAMA MOTA, 2013).

Wennberg, Wiklund e Wright (2011) apontam que os estudantes que já se encontram inseridos no ambiente organizacional estão mais propensos às interações comerciais, do que aqueles indivíduos que somente estão inseridos dentro do contexto universitário, visto que somente a experiência acadêmica não traz as mesmas oportunidades. Logo, um centro de curso empreendedor é aquele que apresenta estudantes engajados em atividade de pesquisa, um centro vinculado à colaboração da indústria e que busca oportunidades fora da Universidade objetivando compartilhar seus resultados com as empresas (GUERRERO, 2015). Assim, formula-se a hipótese: H3: As Informalidades tem um efeito positivo na Universidade Empreendedora. 
A Interação com as Indústrias se refere à visualização da empresa e dos estudantes referente à integração da pesquisa cientifica às indústrias (KALAR e ANTONCIC, 2015; TORODOVIC; MCNAUGHTON e GUILD, 2011). Ipiranga (2008) explica que o contexto da cooperação entre empresas, universidades e governos é formado por questões culturais, vantagens e barreiras políticas e governamentais, formas contratuais e arranjos que se baseiam em distintos objetivos e motivações das instituições envolvidas neste processo.

Neste contexto, a universidade passou a ser visualizada pelas empresas como complemento a pesquisa e ao desenvolvimento organizacional, e a universidade visualizou a empresa como fonte incentivadora de recursos a pesquisa (STAL, 1999; RAPINI, 2006; CRUZ e SEGATTO, 2009; BENEDETTI et al., 2011). As relações entre universidade e empresa fazem parte de um sistema empreendedor que deve interagir de forma a maximizar os benefícios para seus objetivos e, consequentemente, ser o caminho mais rápido para corresponder às necessidades da sociedade, por meio de bens e serviços criados por meio de uma inovação (RIPPER FILHO, 1994; GOMES E PEREIRA, 2005). Destarte, tem-se a hipótese: H4: A Interação das Indústrias tem um efeito positivo na Universidade Empreendedora.

Sherwood e Covin (2008) explicam que uma das melhores formas de se obter Atividades Empreendedoras em uma Universidade é pela transferência de conhecimento e tecnologia entre empresa e universidade, onde a universidade providencia as fontes de tecnologia e pesquisa e a empresa é a receptora do conhecimento. Esta troca de informações depende de alguns fatores culturais determinantes para o empreendedorismo acadêmico.

Estes fatores culturais estão conectados a cultura social econômica do país, considerando o caráter empreendedor; o incentivo a pesquisa universitária por parte da universidade e o incentivo a promoção de novos negócios por parte do governo (TIJSEN, 2006). Esta relação entre universidade, empresa e governo vem sendo estudada por diferentes autores (ETZKOWITZ e LEYDESDORFF, 1999; PARK et. al. 2005; GUNASEKARA 2006; SMITH e LEYDESDORFF, 2014), no qual identificam que quanto maior a parceria, maior a influencia do empreendedorismo acadêmico e a transferência de tecnologia (O’SHEA et al., 2005).

Um dos principais apoiadores e facilitadores da transferência de tecnologia são os centros de cursos das universidades. Esta transferência de tecnologia e conhecimento se dá por meio da experiência dos acadêmicos com as empresas, no qual influenciam o nível de empreendedorismo nos centros de cursos, assim como influenciam a criação de novas start 
up, patentes, licenças e spin offs (O'SHEA et al, 2005; ROTHERMEL et. al. 2007; D'ESTE e PATEL, 2007; RASMUSSEN, MOSEY e WRIGHT, 2014; GUERRERO, 2015).

Porém, há também a influência em atividades mais tradicionais como a participação em eventos científicos, publicação cientifica ou incentivo a prelação de algum tipo de ensino. Portanto, um centro acadêmico tem alta capacidade de encorajar seus estudantes a buscarem por novas oportunidades empreendedoras (KALAR e ANTONCINC, 2015; TORODOVIC; MCNAUGHTON e GUILD, 2011). Assim, levanta-se a hipótese: H5: A Universidade Empreendedora tem um efeito positivo nas Atividades Empreendedoras.

O modelo teórico apresentado na Figura 01 descreve as hipóteses para este estudo.

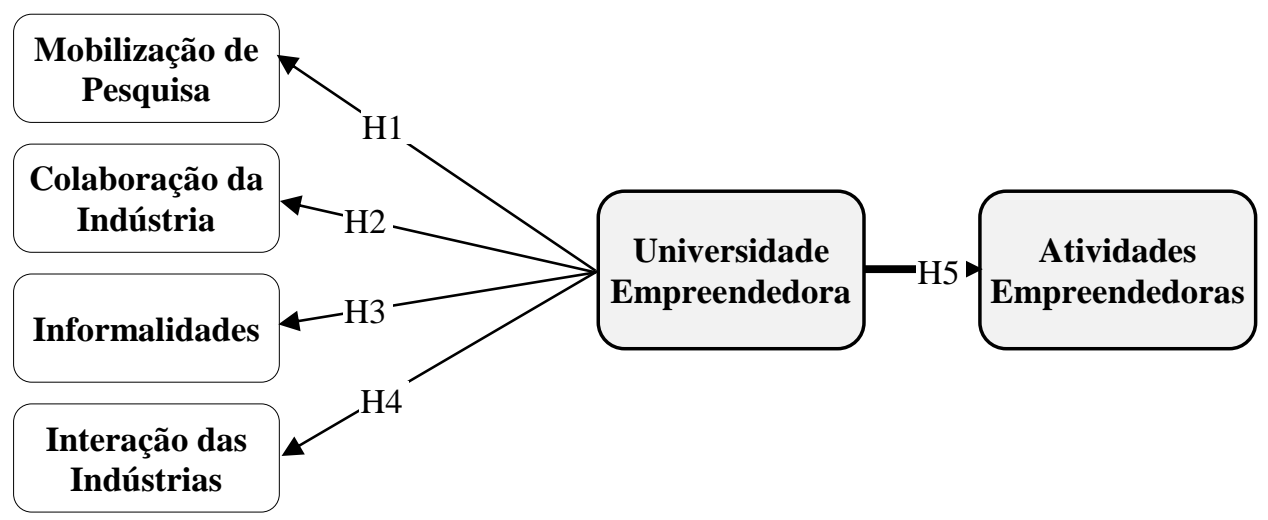

Figura 01 - Modelo proposto para a análise. Fonte: Elaborado pelos autores (2015).

A Universidade Empreendedora é um constructo de segunda ordem composto pela Mobilização de Pesquisa, Colaboração da Indústria, Informalidades e a Interação das Indústrias, que em conjunto, influenciam as Atividades Empreendedoras. O modelo teórico a ser testado é baseado nos estudos de Kalar e Antoncic (2015) e Torodovic, Mcnaughton e Guild (2011), no qual apresenta como base o desenvolvimento do conceito da Universidade Empreendedora.

\section{MÉTODOS E TÉCNICAS DE PESQUISA}

A pesquisa se classifica como quantitativa quanto a abordagem, descritiva e causal, no que tange aos objetivos, survey e de corte transversal (CRESWWLLl, 2010; RICHARDSON, 2012; HAIR Jr et al., 2005). Tendo em conta que o desenvolvimento de novos constructos ou escalas de medida é uma tarefa complexa, seguiu-se a sugestão feita por Prajogo e Sohal (2004) de utilizar constructos pré-testados a partir de estudos empíricos anteriores para 
garantir a validade e confiabilidade. Portanto, os constructos foram mensurados a partir de uma escala chamada ENTRE-U, desenvolvida por Todorovic et. al. (2011).

A escala ENTRE-U foi inicialmente elaborada para ser respondida pelos coordenadores de cada centro de curso, porém Kalar e Antoncic (2015) propuseram em seu estudo que a escala fosse medida por meio da percepção dos estudantes e seu conhecimento referente a orientação empreendedora de seus centros de curso, esta pesquisa seguir o sugerido pelos autores supracitados. O Quadro 1 apresenta as Dimensões e Indicadores utilizados na pesquisa.

Tabela 01: Dimensões e Indicadores.

\begin{tabular}{|c|c|c|}
\hline Indicadores & Variáveis & Autores \\
\hline \multicolumn{3}{|c|}{ Mobilização de Pesquisa } \\
\hline MOBPES1 & $\begin{array}{l}\text { Participação em pesquisas com impactos nas empresas } \\
\text { e sociedade }\end{array}$ & \multirow{6}{*}{ 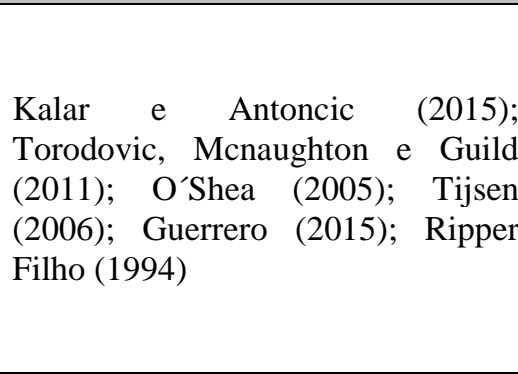 } \\
\hline MOBPES2 & Participação em pesquisas científicas & \\
\hline MOBPES3 & Estimulo da pesquisa pelo centro do curo & \\
\hline MOBPES4 & Contribuição do centro do curso a pesquisa & \\
\hline MOBPES5 & $\begin{array}{l}\begin{array}{l}\text { Participação em pesquisas com profissionais não } \\
\text { acadêmicos }\end{array} \\
\end{array}$ & \\
\hline MOBPES6 & $\begin{array}{l}\text { Expectativa de contribuição cientifica às empresas e } \\
\text { sociedade }\end{array}$ & \\
\hline \multicolumn{3}{|c|}{ Colaboração da Indústria } \\
\hline COLEMP1 & $\begin{array}{l}\text { Centro do curso é conhecido pelas empresas pelo } \\
\text { incentivo à pesquisa }\end{array}$ & \multirow{6}{*}{$\begin{array}{l}\text { Kalar e Antoncic (2015); } \\
\text { Torodovic, Mcnaughton e Guild } \\
\text { (2011); Costa e Cunha (2001); } \\
\text { Segatto e Sbragia (2002); Benedetti } \\
\text { et al. (2011) }\end{array}$} \\
\hline COLEMP2 & Centro do curso conhecido pelo potencial e inovação & \\
\hline COLEMP3 & Estudantes dos cursos que trabalham em altos cargos & \\
\hline COLEMP4 & Centro dos cursos respeitados pelas empresas & \\
\hline COLEMP5 & $\begin{array}{l}\text { Incentivo do centro em atividades de pesquisa nas } \\
\text { empresas }\end{array}$ & \\
\hline COLEMP6 & Centro do curso constrói relacionamento com empresas & \\
\hline \multicolumn{3}{|l|}{ Informalidades } \\
\hline INFORM1 & o identifica oportunidades em empresas & \multirow{6}{*}{$\begin{array}{l}\text { Kalar e Antoncic (2015); } \\
\text { Torodovic, Mcnaughton e Guild } \\
\text { (2011); Rasmussen, Mosey e } \\
\text { Wright (2014),Van Looy et al. } \\
\text { (2011); Ipiranga (2008); Da Gama } \\
\text { Mota (2013); Wennberg, Wiklund e } \\
\text { Wright (2011) }\end{array}$} \\
\hline INFORM2 & Centro do curso busca pesquisa fora da Universidade & \\
\hline INFORM3 & $\begin{array}{l}\text { Centro do curso busca outras fontes além das } \\
\text { governamentais }\end{array}$ & \\
\hline INFORM4 & $\begin{array}{l}\text { Cooperação com organização melhoram as atividades } \\
\text { do centro }\end{array}$ & \\
\hline INFORM5 & $\begin{array}{l}\begin{array}{l}\text { Centro busca } \\
\text { tradicional }\end{array} \\
\end{array}$ & \\
\hline INFORM6 & Centro conhecido por pesquisadores eficientes & \\
\hline \multicolumn{3}{|c|}{ Interação das Indústrias } \\
\hline CONINTEM1 & Fornecimento de pesquisas realizadas as empresas & \multirow{4}{*}{ 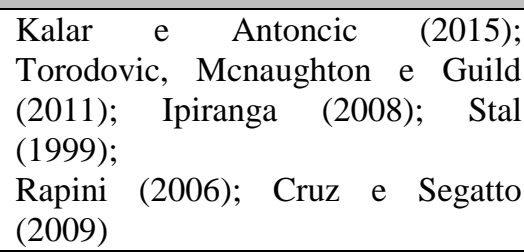 } \\
\hline CONINTEM2 & Contato com empresas para realizar pesquisa & \\
\hline CONINTEM3 & Estudantes do centro estão no mercado de trabalho & \\
\hline CONINTEM4 & $\begin{array}{l}\text { Trabalhar em empresa que se interessa por pesquisa } \\
\text { científica }\end{array}$ & \\
\hline \multicolumn{3}{|c|}{ Atividade Empreendedora } \\
\hline ATIVEMP1 & Pedido de patente & \multirow{2}{*}{$\begin{array}{l}\text { Sherwood e Covin (2008); Tijsen } \\
(2006) ; \quad \text { Park } \quad \text { et al. (2005); }\end{array}$} \\
\hline ATIVEMP2 & Pedido de licença & \\
\hline
\end{tabular}




\begin{tabular}{|c|c|c|}
\hline ATIVEMP3 & Atividade empresarial ou start up & \multirow{5}{*}{$\begin{array}{l}\text { Gunasekara (2006); Smith e } \\
\text { Leydesdorff (2014); Rothermel et } \\
\text { al. (2007); D'Esteandpate (2007); } \\
\text { Rasmussen, Mosey e Wright } \\
\text { (2014), Guerero (2015); Kalar e } \\
\text { Antoncic (2015); Torodovic, } \\
\text { Mcnaughton e Guild (2011) }\end{array}$} \\
\hline ATIVEMP4 & Trabalho científico promovido por empresa & \\
\hline ATIVEMP5 & Promover serviços de pesquisa para empresas & \\
\hline ATIVEMP6 & Promover relatórios de pesquisa para empresas & \\
\hline ATIVEMP7 & $\begin{array}{l}\text { Participação em eventos, workshops, congressos, } \\
\text { fóruns, etc. patrocinados por empresas. }\end{array}$ & \\
\hline
\end{tabular}

Fonte: Elaborado pelos autores (2015).

Os acadêmicos foram solicitados a responder um questionário com escala Likert de 7 pontos (1 - "Discordo Totalmente" e 7 - "Concordo Totalmente"). Os constructos utilizados na pesquisa foram: Mobilização de Pesquisa, que busca de identificar o quão engajado é o estudante em pesquisas científicas influenciadas pelos centros de curso. Colaboração da Indústria, que trada de identificar o reconhecimento dos centros de curso pelas empresas. Informalidades, que descreve os benefícios gerados pelas parcerias de pesquisa entre empresas e a universidade. Interação da Indústria, que trata de identificar o quão engajado é o aluno às empresas. $\mathrm{E}$ as Atividades Empreendedoras, que aponta as atividades empreendedoras exercidas pelos alunos e sua relação com a pesquisa cientifica (TORODOVIC; MCNAUGHTON e GUILD, 2011; KALAR e ANTONCIC, 2015).

A definição da amostra foi intencional, por acessibilidade e conveniência. Este estudo foi conduzido em uma Universidade do Vale do Itajaí, com mais de 50 anos de história, sendo reconhecida e credenciada pelo Ministério da Educação como uma Universidade, no qual apresenta 10.612 alunos. É formada por um instituto que promove parcerias com a iniciativa privada, terceiro setor e poder público, nas áreas de ciências agrárias, ciências biológicas, ciências da saúde, ciências exatas e da terra, ciências humanas, ciências sociais aplicadas, engenharias, linguística, letras e artes.

O procedimento de coleta de dados teve início por meio de envio do questionário online a todos os estudantes de cada centro de curso. O universo de dados foi composto pelos alunos matriculados no $1^{\circ}$ semestre de 2015 . Tabela 01 exemplifica a estrutura da amostra.

Tabela 02: Estrutura da amostra

\begin{tabular}{l|l|l|l|l|l|l|l|l}
\hline Centros de cursos & CCSA & CCE & CCEN & CCHC & CCJ & CCT & CCS & TOTAL \\
\hline Número total de alunos & 1598 & 462 & 1033 & 828 & 1299 & 2969 & 2482 & 10671 \\
\hline Número da Amostra & 181 & 94 & 24 & 14 & 162 & 87 & 86 & $\mathbf{6 4 8}$ \\
\hline
\end{tabular}

Fonte: dados da pesquisa (2015).

Os centros de curso são divididos entre Centro de Ciências Sociais Aplicadas (CCSA), Centro de Ciências Exatas e Naturais (CCEN), Centro de Ciências da Educação, Artes e Letras (CCEAL), Centro de Ciências Humanas e da Comunicação (CCHC), Centro de 
Ciências Jurídicas (CCJ), Centro de Ciências Tecnológicas (CCT) e Centro de Ciências da Saúde (CCS).

Os dados foram analisados por meio de Modelagem de Equações Estruturais (MEE) com o intuito de averiguar a causalidade entre os constructos. Esta técnica estatística é projetada para a situação em que as ligações entre as variáveis observadas e latentes são desconhecidas ou incertas. Tipicamente, o investigador pretende identificar o número mínimo de fatores que representam as covariações entre as variáveis observadas (BYRNE, 2013).

Os índices de qualidade de ajustamento são utilizados para avaliar os resultados sob três perspectivas: ajuste global, ajuste comparado a um modelo básico e parcimônia do modelo (HAIR Jr. et al., 2005). A confiabilidade de cada constructo foi calculada individualmente. Para tal, adotou-se o Alfa de Cronbach, sendo aceitos valores de 0,5 a 0,7, apesar de valores abaixo destes serem aceitáveis em pesquisas de natureza exploratória.

Hair, Jr. et al. (2005) indicam limitações no coeficiente Alfa de Cronbach (AC). Para os autores, o cálculo da confiabilidade por meio do Alfa de Cronbach não considera os erros nos indicadores. Devido a este fato foi utilizado a Confiabilidade Composta (CC) e a Variância Média Extraída (Average Variance Extracted AVE). A CC é uma medida de consistência interna dos itens, sugerem-se valores maiores que 0,70. A AVE representa uma medida de confiabilidade que indica a quantidade geral de variância nos indicadores explicada pelo constructo latente. Recomendam-se valores superiores a 0,5 para um constructo (HAIR, Jr. et al., 2005). Para o tratamento dos dados foram utilizados os programas SPSS ${ }^{\circledR}$ (Statistical Package for the Social Sciences) versão 22 e $\operatorname{AMOS}^{\circledR}$ versão 20.

\section{APRESENTAÇÃO E ANÁLISE DOS RESULTADOS}

Nesta etapa serão apresentados e analisados os dados obtidos para a pesquisa, iniciando com a apresentação dos indicadores do Alfa de Cronbach, Confiabilidade Composta (CC) e Variância Média Extraída (Average Variance Extracted AVE). Posteriormente, será realizada a MEE para verificar a relação entre os indicadores do modelo. A Tabela 02 apresenta os índices de confiabilidade do modelo de mensuração.

Tabela 03: Indicadores de confiabilidade

\begin{tabular}{l|l|l|l|l}
\hline Constructos & A.C & C.C & AVE & R Square $\left(\mathbf{R}^{2}\right)$ \\
\hline Mobilização de Pesquisa & 0,88 & 0,89 & 0,60 & 0,72 \\
\hline Colaboração da Indústria & 0,90 & 0,90 & 0,60 & 0,88 \\
\hline Informalidades & 0,92 & 0,92 & 0,66 & 0,85 \\
\hline Interação das Indústrias & 0,65 & 0,67 & 0,37 & 0,40 \\
\hline
\end{tabular}




\begin{tabular}{l|l|l|l|l}
\hline Atividade Empreendedora & 0,90 & 0,92 & 0,66 & 0,08 \\
\hline
\end{tabular}

Fonte: dados da pesquisa (2015).

Conforme a Tabela 02 constatou-se que as dimensões foram consideradas fidedignas, uma vez que atingiram valores superiores ao recomendado (HAIR, Jr. et al., 2005). A Tabela 03 apresenta as medidas de qualidade de ajuste absoluto e os índices de ajuste incremental do modelo empírico de uma cultura organizacional que suporta a inovação.

Tabela 04: Índices de ajustes para o modelo

\begin{tabular}{l|l|l}
\hline Medidas de Ajuste & Nível Aceitável & Nível Encontrado \\
\hline GL & - & 294 \\
\hline$\chi^{2}$ e $\mathrm{p}$ & $-(\mathrm{p}<0,000)$ & $1707,037(\mathrm{p}<0,000)$ \\
\hline$\chi^{2} / \mathrm{GL}$ & $\leq 5$ & 5,8 \\
\hline GFI & $>0,90$ & 0,822 \\
\hline AGFI & $>0,90$ & 0,788 \\
\hline RMR & $<0,10$ & 0,071 \\
\hline RMSEA & 0,05 a 0,08 & 0,086 \\
\hline NFI & $>0,90$ & 0,864 \\
\hline TLI & $>0,90$ & 0,872 \\
\hline CFI & $>0,90$ & 0,884 \\
\hline & Fonte: dados da pesquisa (2015).
\end{tabular}

As medidas de qualidade de ajuste absoluto apresentaram $\chi^{2}$ da razão de verossimilhança de 1707,037, para 294 graus de liberdade (GL), sendo estatisticamente significantes no nível de $0,000(p<0,05)$. Quando analisada a qualidade do modelo $\chi^{2}$ sobre GL, obtêm-se 5,8. O índice de qualidade de ajuste (GFI) foi de 0,822, cujos valores variam de 0 (ajuste pobre) a 1,0 (ajuste perfeito). O valor da raiz do resíduo quadrático médio (RMSEA) atingiu o valor de 0,086, ficando próximo do limite aceitável (KLINE, 2005; HAIR, Jr. et al., 2005).

Em seguida analisaram-se os índices de ajuste incremental. O índice de Tucker-Lewis (TLI) teve um valor de 0,872 e o índice de ajuste comparativo (CFI) foi de 0,884, ou seja, todos os índices de ajuste incremental ficaram próximos dos parâmetros de referência $(0,90)$. Com base nesses resultados pode-se considerar que o modelo obteve um bom ajuste (HAIR, Jr. et al., 2005).

Considerando que não foram encontrados estudos anteriores usando o modelo proposto no contexto nacional, acarretando assim na ausência de evidências empíricas que possibilitem comparações efetivas, entende-se que o modelo, ainda que não tenha 


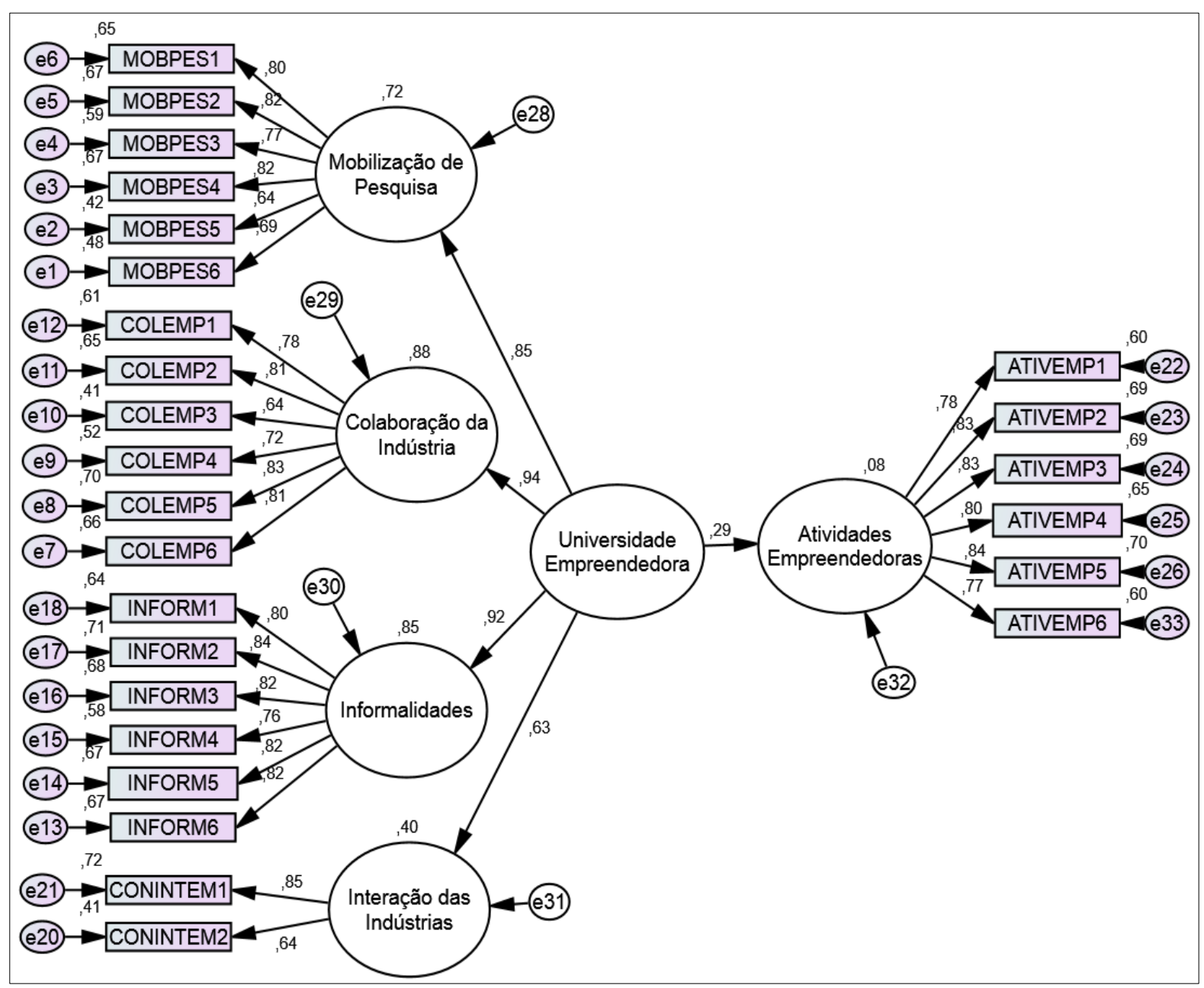

demonstrado valores satisfatórios em todas as medidas de ajuste, pode ser aperfeiçoado com novos estudos empíricos, por conseguinte, não o invalidando. A Figura 02 apresenta o modelo estrutural final.

Figura 02 - Modelo Teórico dos constructos da Universidade empreendedora que influenciam as atividades empreendedoras Fonte: dados da pesquisa (2015).

A partir da Figura 02 verificam-se as cargas fatoriais padronizadas e o $\mathrm{R}^{2}$ para a análise do modelo proposto. Optou-se pela exclusão da variável "CONINTEM3” (Estudantes do centro estão no mercado de trabalho) e "CONINTEM4" (Trabalhar em empresa que se interessa por pesquisa científica) da dimensão Interação das Industrias, uma vez que apresentou carga padronizada menor que 0,60. As demais cargas padronizadas foram superiores 0,60, em conformidade com Kline (2005).

É possível verificar que o $\mathrm{R}^{2}$ foi de 0,88 para a dimensão Colaboração da Indústria, ou seja, $88 \%$ da variância observada na variável é explicada pelas variáveis que compõem o 
modelo, o $\mathrm{R}^{2}$ foi de $85 \%$ para Informalidades, $72 \%$ para Mobilização de Pesquisa, $40 \%$ para Interação das Indústrias e $29 \%$ para Atividades Empreendedoras. O $\mathrm{R}^{2}$ das dimensões que formam a dimensão Universidade Empreendedora apresentou, de forma geral coeficientes altos, o que confere boa explicação da variância pelas variáveis independentes. Já o $\mathrm{R}^{2}$ das variáveis Atividades Empreendedoras que é influenciado pela Universidade Empreendedora apresentou $\mathrm{R}^{2}$ de 0,08 , indicando a baixa influência da Atividades Empreendedoras na Universidade estudada.

Após a verificação do ajustamento do modelo, foram analisadas as cargas fatoriais padronizadas e os respectivos $t$-values a fim de testar as hipóteses, conforme mostra a Tabela 04.

Tabela 05 - Coeficientes padronizados, significâncias e hipóteses do modelo proposto.

\begin{tabular}{c|c|c|c|c|c|c|l}
\hline \multicolumn{2}{l|}{ CAMINHOS ESTRUTURAIS } & $\begin{array}{l}\text { Erro } \\
\text { Padrão }\end{array}$ & t-values & $\boldsymbol{p}$ & $\begin{array}{l}\text { Coef. } \\
\text { Padronizado }\end{array}$ & Hipóteses \\
\hline $\begin{array}{c}\text { Mobilização de } \\
\text { Pesquisa }\end{array}$ & $\leftarrow$ & $\begin{array}{c}\text { Universidade } \\
\text { Empreendedora }\end{array}$ & 0,061 & 17,221 & 0,00 & 0,851 & H1 - (Suportada) \\
\hline $\begin{array}{c}\text { Colaboração da } \\
\text { Indústria }\end{array}$ & $\leftarrow$ & $\begin{array}{c}\text { Universidade } \\
\text { Empreendedora }\end{array}$ & 0,059 & 22,419 & 0,00 & 0,936 & H2 - (Suportada) \\
\hline Informalidades & $\leftarrow$ & $\begin{array}{c}\text { Universidade } \\
\text { Empreendedora }\end{array}$ & 0,060 & 22,358 & 0,00 & 0,921 & $\mathbf{H 3}$ - (Suportada) \\
\hline Interação das Indústrias & $\leftarrow$ & $\begin{array}{c}\text { Universidade } \\
\text { Empreendedora }\end{array}$ & 0,073 & 14,104 & 0,00 & 0,631 & $\mathbf{H 4}$ - (Suportada) \\
\hline $\begin{array}{c}\text { Atividades } \\
\text { Empreendedoras }\end{array}$ & $\leftarrow$ & $\begin{array}{c}\text { Universidade } \\
\text { Empreendedora }\end{array}$ & 0,059 & 6,153 & 0,00 & 0,285 & $\mathbf{H 5}$ - (Suportada) \\
\hline
\end{tabular}

Fonte: Dados da pesquisa.

Em termos de indicadores para a aceitação das hipóteses, os t-values necessitam ser superiores a 1,96 (para aceitação tolerável); o ideal é acima de 2,58, para acatar significância apropriada (HAIR, Jr. et al., 2005). Conforme a Tabela 04, os resultados dos t-values foram altamente satisfatórios apenas para a relação dos construtos da universidade empreendedora que influenciam as atividades empreendedoras.

Com base na Tabela 04, no que tange a Mobilização de Pesquisa que apoiam a Universidade Empreendedora a partir do modelo de Kalar e Antoncic (2015) e Torodovic, Mcnaughton e Guild (2011), constata-se uma relação positiva, com um bom índice de significância, ou seja, o caminho (path) entre Mobilização de Pesquisa <Universidade Empreendedora foi de $\lambda=0,851, \mathrm{p}<0,00$, com isso, aceita-se a H1. Ficou evidenciado que no modelo testado a Mobilização de Pesquisa influencia a Universidade Empreendedora.

A mobilização de pesquisa se refere ao encorajamento da Universidade a seus alunos em se engajarem a cultura empreendedora, com o objetivo do desenvolvimento econômico e social, contribuindo para o surgimento de novos negócios (O’SHEA et al., 2005; TIJSEN 
2006; GUERRERO et. al., 2015). É importante a Universidade apresentar mobilização a pesquisa, pois estas vantagens estão ligadas aos objetivos básicos da Universidade e da empresa, ou seja, a universidade continua contribuindo para a sua missão de formar recursos humanos e a empresa notar nela uma contribuição direta ou indireta para sua lucratividade (RIPPER FILHO, 1994).

Percebeu-se uma relação positiva entre a Colaboração das Indústrias «Universidade Empreendedora $(\lambda=0,936, \mathrm{p}<0,00)$, com este resultado, aceita-se a H2. Apesar de no Brasil esta aproximação entre universidade e empresa ser vista de forma precária (COSTA e CUNHA, 2001; SEGATTO e SBRAGIA, 2002; BENEDETTI et al., 2011), para Segatto e Sbragia (2002) o fundo governamental de apoio à pesquisa pode ser um dos principais facilitadores da parceria entre empresa e indústria, uma vez iniciada a cooperação, as divergências entre as duas esferas são minimizadas pelo estreitamento do relacionamento, o que contribui para a velocidade da execução do projeto de desenvolvimento de uma nova tecnologia (BENEDETTI et al., 2011).

A relação entre Informalidade $\leftarrow$ Universidade Empreendedora é de $\lambda=0,921, \mathrm{p}<$ 0,00, neste sentido, aceita-se a H3. As informalidades estão conectadas ao nível empreendedor da Universidade na busca por parceiros comerciais (VAN LOOY et al., 2011; RASMUSSEN, MOSEY e WRIGHT, 2014), ou incentivos governamentais por meio da CAPES e CNPq, com a finalidade de incentivar a parcerias entre empresas e universidades (IPIRANGA, 2008; DA GAMA MOTA, 2013). A Universidade que não demonstra estes interesses acabada desencorajando os estudantes a buscarem por novas oportunidades (RASMUSSENA, MOSEY e WRIGHT, 2014). Pois com este envolvimento, estudantes que já se encontram inseridos no ambiente organizacional estão mais propensos a interações comerciais, (WENNBERG, WIKLUND e WRIGHT, 2011). Logo, a universidade empreendedora é aquela que apresenta estudantes engajados em atividade de pesquisa, vinculado a colaboração da indústria e que busca oportunidades fora da Universidade objetivando compartilhar seus resultados com as empresas (GUERRERO, 2015)

A Interação das Indústrias apresentou relação positiva e significativa com a Universidade Empreendedora, $(\lambda=0,631, \mathrm{p}<0,631)$, assim aceita-se a H4. A visualização da Universidade como empreendedora deve interatuar de forma a maximizar os benefícios para seus objetivos e, consequentemente, ser o caminho mais rápido para corresponder às necessidades da sociedade, por meio de bens e serviços criados por meio de uma inovação (RIPPER FILHO, 1994; GOMES e PEREIRA, 2005). 
Por fim, aceita-se a H5, a Universidade Empreendedora influencia as Atividades empreendedoras com coeficiente padronizado de $\lambda=0,285, \mathrm{p}<0,00$. A relação entre Universidade Empreendedora e Atividades Empreendedoras pode, portanto, ser considerada positiva, mas com intensidade relativamente fraca, uma vez que o coeficiente de determinação $\left(\mathrm{R}^{2}\right)$ obtido foi de aproximadamente $8 \%$.

Deste modo, podem haver outras dimensões que, além da Universidade Empreendedora, estão influenciando as Atividades Empreendedoras na amostra da Universidade estudada. A adoção de atividades empreendedoras por uma Universidade depende de fatores culturais, conectados a cultura sócia econômica do país, considerando o seu caráter empreendedor. Portanto, conforme afirma Tijsen (2006) deve-se haver um incentivo a pesquisa universitária partindo da universidade e o incentivo a promoção de novos negócios partindo do governo.

Sendo o centro dos cursos um dos principais apoiadores e facilitadores da transferência de tecnologia, conforme afirmam os autores: O'shea et. al (2005), Rothermel et. al. (2007); D'este e patel (2007); Rasmussen, Mosey e Wright (2014) e Guerrero (2015) a transferência de tecnologia e conhecimento acontece via a experiência dos acadêmicos com as empresas, no que consecutivamente influenciam o nível de empreendedorismo nos centros de cursos, assim como influenciam a criação de novas start up, patentes, licenças e spin offs .

Além de que outro fator de influência ao empreendedorismo acadêmico e a transferência de tecnologia é a relação entre universidade, empresa e governo, no qual influenciam atividades empreendedoras (ETZKOWITZ e LEYDESDORFF, 1999; PARK et al. 2005; GUNASEKARA 2006; SMITH e LEYDESDORFF, 2014), deixando de lado atividades mais tradicionais como a participação em eventos científicos, publicação cientifica ou incentivo a prelação de algum tipo de ensino (KALAR e ANTONCINC, 2015).

Desta forma, as atividades empreendedoras são influenciadas pelos constructos da Universidade empreendedora, porém a Universidade estudada, assim como seus centros acadêmicos, apresenta baixos índices de orientação ao empreendedorismo, desfavorecendo a influência de suas atividades empreendedoras.

\section{CONCLUSÃO}

O objetivo deste estudo foi analisar a influência da Universidade Empreendedora formada pelas dimensões de Mobilização de Pesquisa, Colaboração da Indústria, 
Informalidades e Interação das Indústrias nas Atividades Empreendedoras no contexto de uma Universidade de Santa Catarina.

Os resultados mostram que a Universidade Empreendedora pode ser abordada utilizando as dimensões de primeira ordem Mobilização de Pesquisa, Colaboração da Indústria, Informalidades e Interação das Indústrias. Portanto, a Mobilização de Pesquisa, que se refere ao incentivo dos centros do curso aos alunos referente às pesquisas científicas com impacto nas empresas e sociedade, é importante para que uma universidade seja empreendedora.

Outro importante influenciador é a colaboração da indústria, que parte da busca dos centros de cursos por incentivos a inovação e ao relacionamento junto às empresas, bem como a busca informal da universidade pela colaboração externa, seja ela com as empresas ou o governo. Além, da clara visualização do aluno perante estes incentivos, que fazem com que a interação com as indústrias ocorra mais facilmente, tornando assim a universidade cada vez mais empreendedora.

Já as informalidades que se diz respeito à busca da universidade por parceiros comerciais, também se encontra como um importante influenciador; bem como a interação das indústrias, no qual evidencia que a interação com parceiros comerciais, pode influenciar no suprimento das necessidades de uma sociedade, sendo assim este um importante influenciador da universidade empreendedora.

Por fim, a pesquisa evidenciou que a Universidade Empreendedora tem influencia nas Atividades Empreendedoras. Corroborando que quanto mais os indicadores citados acima forem reforçados, maior será a transferência de conhecimento entre empresa e universidade, desenvolvendo assim a criação de novos conhecimentos e oportunidades em sociedade, como novas invenções, patentes, licenças, spin offs e etc. Porém, conforme verificado neste estudo, a universidade estudada apresenta baixa influencia dos indicadores da universidade empreendedora em suas atividades empreendedoras, evidenciando a falta do empreendedorismo universitário.

O modelo estudado fornece uma estrutura para pensar sobre o empreendedorismo universitário, promovendo os indicadores que influenciam na transferência de conhecimento e tecnologia entre empresa e universidade. Verificou-se que os indicadores são fundamentais para que ocorram atividades empreendedoras.

Com este estudo surgem oportunidades de pesquisas futuras, como a aplicação do modelo em diferentes universidades, bem como a verificar a intensidade dos indicadores 
empreendedores. A replicação desta pesquisa contribui também para o desenvolvimento desta área de estudo, tendo em vista a escassez de trabalhos empíricos sobre empreendedorismo universitário e sua intenção com o desenvolvimento nas universidades.

\section{REFERÊNCIAS}

AUDRETSCH, D. B. From the entrepreneurial university to the university for the entrepreneurial society. The Journal of Technology Transfer, New York, v. 39, n. 3, p. 313$321,2014$.

BYRNE, B. M. Structural equation modeling with AMOS: Basic concepts, applications, and programming. Abingdon: Routledge, 2013.

COVIN, J. G.; SLEVIN, D. P. Strategic management of small firms in hostile and benign environments. Strategic Management Journal, Hoboken, v. 10, n. 1, p. 75, 1989.

CRESWELL, J. W. Projeto de pesquisa métodos qualitativo, quantitativo e misto. In: Projeto de pesquisa métodos qualitativo, quantitativo e misto. Porto Alegre: Artmed, 2010.

DA GAMA MOTA, T. L. N. Interação universidade-empresa na sociedade do conhecimento: reflexões e realidade. Ciência da Informação, Brasília, v. 28, n. 1, 2013.

DE ANDRADE MARTINS, G.; THEÓPHILO, C. R. Metodologia da investigação científica para ciências sociais aplicadas. Porto Alegre: Atlas, 2007.

DEL-VECCHIO, R. R.; BRITTO, J.; OLIVEIRA, B. F. Patterns of university-industry interactions in Brazil: an exploratory analysis using the instrumental of graph theory. Quality \& Quantity, New York, v. 48, n. 4, p. 1867-1892, 2014.

D'ESTE, P.; PATEL, P. University-industry linkages in the UK: What are the factors underlying the variety of interactions with industry? Research policy, Amsterdam, v. 36, n. 9, p. 1295-1313, 2007.

ETZKOWITZ, H.; LEYDESDORFF, L. The future location of research and technology transfer. The Journal of Technology Transfer, New York, v. 24, n. 2-3, p. 111-123, 1999.

ETZKOWITZ, H. et al. The future of the university and the university of the future: evolution of ivory tower to entrepreneurial paradigm. Research policy, Amsterdam, v. 29, n. 2, p. 313$330,2000$.

ETZKOWITZ, H.; ZHOU, C. Introduction to special issue Building the entrepreneurial university: a global perspective. Science and Public Policy, Oxford, v. 35, n. 9, p. 627-635, 2008 .

ETZKOWITZ, H. Anatomy of the entrepreneurial university. Social Science Information, Thousand Oaks, v. 52, n. 3, p. 486-511, 2013. 
ETZKOWITZ, H. The norms of entrepreneurial science: cognitive effects of the new university-industry linkages. Research Policy, Amsterdam, v. 27, n. 8, p. 823-833, 1998.

GUERRERO, M.; URBANO, D. The development of an entrepreneurial university. The Journal Of Technology Transfer, New York, v. 37, n. 1, p. 43-74, 2012.

GUERRERO, M.; CUNNINGHAM, J. A.; URBANO, D. Economic impact of entrepreneurial universities' activities: An exploratory study of the United Kingdom. Research Policy, Amsterdam, v. 44, n. 3, abr. 2015.

GUNASEKARA, C. Reframing the role of universities in the development of regional innovation systems. The Journal of Technology Transfer, New York, v. 31, n. 1, p. 101$113,2006$.

HAIR JR, J. et al. Fundamentos de métodos de pesquisa em administração. Porto Alegre: Bookman, 2005.

KALAR, B.; ANTONCIC, B. The entrepreneurial university, academic activities and technology and knowledge transfer in four European countries. Technovation, Amsterdam, v. 36, p. 1-11, 2015.

LEYDESDORFF, L.; MEYER, M. Triple Helix indicators of knowledge-based innovation systems: Introduction to the special issue. Research policy, Amsterdam, v. 35, n. 10, p. 14411449, 2006.

MANCINI, R. F.; LORENZO, H. C. Potencialidades e barreiras à cooperação universidade, empresa e governo: o caso das micro e pequenas empresas do segmento médico odontológico do município de Araraquara. In: ENCONTRO NACIONAL DE ENGENHARIA DE PRODUÇÃO, 26., Fortaleza. Anais... Fortaleza: Enegep, 2006. CDROM.

MALHOTRA, N. K. Pesquisa de marketing: uma orientação aplicada. Porto Alegre: Bookman, 2012.

PARK, H. W.; HONG, H. D.; LEYDESDORFF, L. A comparison of the knowledge-based innovation systems in the economies of South Korea and the Netherlands using Triple Helix indicators. Scientometrics, New York, v. 65, n. 1, p. 3-27, 2005.

PRAJOGO, D. I.; SOHAL, A. S. The multidimensionality of TQM practices in determining quality and innovation performance - an empirical examination. Technovation, Amsterdam, v. 24, n. 6, p. 443-453, 2004.

PHILPOTT, K. et al. The entrepreneurial university: Examining the underlying academic tensions. Technovation, Amsterdam, v. 31, n. 4, p. 161-170, 2011.

O'SHEA, R. P. et al. Entrepreneurial orientation, technology transfer and spinoff performance of US universities. Research policy, Amsterdam, v. 34, n. 7, p. 994-1009, 2005. 
RAPINI, M. S. Interação universidade-empresa no Brasil: evidências do Diretório dos Grupos de Pesquisa do CNPq. Estudos Econômicos, São Paulo, v. 37, n. 1, p. 211-233, 2007.

RASMUSSEN, E.; MOSEY, S.; WRIGHT, M. The influence of university departments on the evolution of entrepreneurial competencies in spin-off ventures. Research Policy, Amsterdam, v. 43, n.1, p. 92-106, 2014.

RIPPER FILHO, J. E. Ciência e tecnologia: para quê? como? In: VOGT, C.; STAL, E. Ciência e Tecnologia: alicerces do desenvolvimento. São Paulo: CNPq, 1994.

RICHARDSON, R. J. et al. Pesquisa social: métodos e técnicas. São Paulo: Atlas, 2012.

SBRAGIA, R. et al. Como vencer esse desafio empresarial. São Paulo: Clio Editora, 2006.

SHANE, S. Selling university technology: patterns from MIT. Management Science, Catonsville, v. 48, n. 1, p. 122-137, 2002.

SMITH, H. L.; LEYDESDORFF, L. The Triple Helix in the context of global change: dynamics and challenges. Prometheus, Abingdon, v. 32, n. 4, p. 321-336, 2014.

TODOROVIC, Z. W.; MCNAUGHTON, R. B.; GUILD, P. ENTRE-U: An entrepreneurial orientation scale for universities. Technovation, Amsterdam, v. 31, n. 2, p. 128-137, 2011.

IPIRANGA, A. S. R.; FREITAS, A. A. F.; PAIVA, T. A. O empreendedorismo acadêmico no contexto da interação universidade-empresa-governo. Cad. EBAPE. BR, Rio de Janeiro, v. 8, n. 4, p. 687-693, 2010.

VAN LOOY, B. et al. Entrepreneurial effectiveness of European universities: An empirical assessment of antecedents and trade-offs. Research Policy, Amsterdam, v. 40, n. 4, p. 553 $564,2011$.

WENNBERG, K.; WIKLUND, J.; WRIGHT, M. The effectiveness of university knowledge spillovers: Performance differences between university spinoffs and corporate spinoffs. Research Policy, Amsterdam, v. 40, n. 8, p. 1128-1143, 2011.

WRIGHT, M. et al. University spin-out companies and venture capital. Research policy, Amsterdam, v. 35, n. 4, p. 481-501, 2006. 